\title{
Caracterização nutricional do nim em plantios no Brasil
}

\author{
Edinelson J. M. Neves ${ }^{1}$, Carlos B. Reissmann' ${ }^{2}$, Renato A. Dedecek ${ }^{1}$ \& Antônio A. Carpanezzi ${ }^{1}$
}

\begin{abstract}
RESUMO
No Brasil são incipientes os estudos sobre os aspectos nutricionais do cultivo do nim. Propôs-se, neste trabalho, determinar os teores foliares de macronutrientes em plantios de nim estabelecidos em oito Estados do Brasil e correlacioná-los com os nutrientes disponíveis na camada de 0-20 cm de profundidade dos solos sob os plantios amostrados. De cada plantio foram coletadas folhas de nove árvores das partes média/superior e média/inferior da copa, as quais foram misturadas, formando duas amostras compostas denominadas folhas da copa média/superior (FCMS) e folhas da copa média/inferior (FCMI). Para análise de variância dos teores foliares de nutrientes utilizou-se o delineamento inteiramente casualizado, com decomposição total dos graus de liberdade visando ao efeito local de plantio, idade de plantio e posição na copa, sendo a comparação de médias realizada pelo Teste de Duncan $(p \leq 0,05)$. A amostragem foliar nas árvores de nim depende, quando feita na posição mediana subdividida em FCMS e FCMI, entre outros fatores, da mobilidade do nutriente e da condição de luminosidade do plantio. Os teores foliares de $\mathrm{Ca}$ apresentam indícios de ser o nim uma espécie calcícola. O nim é eficiente na absorção de N, P e Ca. O K do solo apresenta correlação positiva com o K das FCMI.
\end{abstract}

Palavras-chave: Azadirachta indica A. Juss., macronutrientes da planta, relação solo-planta

\section{Nutritional characterization of neem plantations in Brazil}

\begin{abstract}
In Brazil, there are very few studies about nutritional status of neem. This study aimed to determine the contents of macronutrients in leaf in neem plantations established in different locations in eight states of Brazil, and perform correlations with the available nutrients in the respective soils under the sampled plantation. From each plantation the leaves were collected from nine trees, from the middle superior and middle inferior part of the crown. Each part of the crown of the nine trees were mixed, forming two composite samples and were nominated as leaves from the middle superior crown (FCMS), and middle inferior crown (FCMI). At the time of leaf sampling, the soil was collected in $0-20 \mathrm{~cm}$ depth. For variance analysis of the nutrient contents of leaf, a completely randomized design was used, applying total decomposition of the degrees of freedom for stand localization, stand age and crown position effect, being the mean comparisons performed by the Duncan test $(p \leq 0,05)$. Additional correlation and cluster analysis were applied integrating soil data. The leaf sampling of neem trees, when realized in the middle of the crown subdivided in FCMS and FCMI depends, among other factors, on the nutrient mobility and on the light conditions of the stand. The leaf contents of Ca show indications that the neem plant could be a calcicole plant, and efficient in $\mathrm{N}$ and $\mathrm{P}$ absorption. The soil $\mathrm{K}$ content is positively correlated with the $\mathrm{K}$ content of the FCMI position.
\end{abstract}

Key words: Azadirachta indica A. Juss., plant macronutrients, soil-plant relationship 


\section{INTRODUÇÃO}

O nim (Azadirachta indica A. Juss., Meliaceae) é nativo da India e Myanmar (antiga Burma), onde cresce em florestas de regiões tropicais e subtropicais. Nessas regiões o nim se apresenta como árvore de crescimento rápido e perenifólia (Maithani et al., 2011). É uma planta utilizada há mais de 4.000 anos em virtude de suas propriedades medicinais apresentadas por seus frutos, sementes, folhas, cascas e raízes conterem compostos com comprovada ação antisséptica, anti-inflamatória e antifúngicas, entre outros (Girish \& Bhat, 2008). Por isto, em países onde a espécie é nativa o extrato das folhas do nim é usado no tratamento de úlcera, de diabete e em procedimentos periodônticos (Pankaj et al., 2011).

$\mathrm{Na}$ Índia o nim é conhecido por sua tolerância à seca pois cresce em áreas com regime de chuva que varia de 400 a $1200 \mathrm{~mm}$ e em diferentes tipos de solo (Maithani et al., 2011) porém esses autores mencionam que o nim apresenta melhor crescimento quando cultivado em solos profundos, arenosos, bem drenados e com pH entre 6,2 e 7,0.

No Brasil a condução de plantios com nim em regiões dos biomas Cerrado, Caatinga, Mata Atlântica e Amazônia impõe mudanças no manejo e na adubação adotado. Isto requer que nesses biomas o cultivo do nim seja conduzido preferencialmente em solos com características edafoclimáticas similares às mencionadas por Maithani et al. (2011).

Estudos sobre a nutrição de espécies florestais para diferentes fins vêm sendo utilizados para melhor compreensão da relação solo-planta. Neste contexto, a análise foliar de material coletado em diferentes partes da copa é uma ferramenta que possibilita identificar e corrigir possíveis deficiências minerais, advindas do solo e/ou de adubações feitas.

Objetivou-se, com este trabalho, caracterizar os teores foliares de nitrogênio $(\mathrm{N})$, fósforo $(\mathrm{P})$, potássio $(\mathrm{K})$, cálcio (Ca) e magnésio $(\mathrm{Mg})$ em plantios estabelecidos com diferentes idades e tipos de solo, em vários municípios do Brasil e correlacionar os teores desses elementos minerais, disponíveis nas folhas em duas posições da copa, com os disponíveis no solo sob os mencionados plantios.

\section{MATERIAL E MÉTODOS}

Na condução deste trabalho foram utilizadas árvores de nim plantadas em estações experimentais do IAPAR e em propriedades rurais localizadas em municípios de diferentes estados do Brasil. Na Tabela 1 são mencionadas, de acordo com o local de plantio, as coordenadas geográficas, altitude a nível do mar e tipo de clima, segundo a classificação de Köppen, respectivamente.

Em cada plantio foram selecionadas nove árvores de bom crescimento para a coleta de folhas. As folhas foram coletadas das seguintes partes da copa: 1) parte média/superior e 2) parte média/inferior. As folhas coletadas de cada árvore/parte da copa foram misturadas formando, de cada plantio, duas amostras compostas denominadas folhas da copa média/superior e folhas da copa média/inferior doravante denominadas FCMS e FCMI, respectivamente.

As amostras foram colocadas em estufa a $60^{\circ} \mathrm{C}$, até peso constante; posteriormente, foram moídas e encaminhadas ao Laboratório de Solos e Nutrição de Plantas da Embrapa Florestas para determinação dos teores de N, P, K, Ca e Mg, segundo metodologia proposta por Nogueira \& Souza (2005).

Por ocasião da coleta de folhas foram tomadas amostras de solo de cada plantio na camada de $0-20 \mathrm{~cm}$ de profundidade; para tanto foram marcados, em cada plantio, cinco pontos de coleta na forma de X, para obtenção de uma amostra composta; as análises químicas foram feitas segundo Nogueira \& Souza (2005). Os valores obtidos são apresentados na Tabela 2.

Os locais e tipos de solo usados nos diferentes estados do Brasil são apresentados na Tabela 3

Depois de verificadas a homogeneidade e a normalidade da variância residual foi realizada, para aferição dos teores foliares de nutrientes, a análise de variância considerando-se o delineamento inteiramente casualizado com decomposição total dos graus de liberdade para o efeito local de plantio, idade de plantio e posição na copa.

A análise estatística e a interpretação dos resultados foram realizadas pelo teste de Duncan $(\mathrm{p} \leq 0,05)$. As análises foram feitas usando-se o sistema SAS - Statistical Analysis System, versão 8.2 , através do procedimento GLM.

\section{RESUlTADOS E DISCUSSÃO}

A análise estatística detectou diferenças significativas pelo Teste de Duncan $(p \leq 0,05)$ para os teores foliares médios de $\mathrm{N}, \mathrm{P}, \mathrm{K}, \mathrm{Ca}$ e $\mathrm{Mg}$, entre local de plantio e posição na copa (Tabela 4); quanto à idade de plantio os resultados obtidos

Tabela 1. Local de plantio, coordenadas geográficas, altitude a nível do mar e tipo de clima das áreas com árvores de nim usadas para amostragem foliar

\begin{tabular}{|c|c|c|c|}
\hline Local de plantio & Coordenadas geográficas & Altitude em nível do mar (m) & Tipo de clima \\
\hline Estação do IAPAR em Londrina/PR & $23^{\circ} 21^{\prime} 18^{\prime \prime}$ S e $51^{\circ} 09^{\prime} 45^{\prime \prime} \mathrm{W}$ & 568 & Cfa \\
\hline Estação do IAPAR em Xambrê/PR & $23^{\circ} 44^{\prime} 02^{\prime \prime}$ S e $53^{\circ} 29^{\prime} 27^{\prime \prime} \mathrm{W}$ & 382 & Cfa \\
\hline Jales/SP & $20^{\circ} 16^{\prime} 05^{\prime \prime}$ S e $50^{\circ} 32^{\prime} 55^{\prime \prime} \mathrm{W}$ & 490 & Aw \\
\hline Ibirá/SP & $21^{\circ} 04^{\prime} 50^{\prime \prime}$ S e $49^{\circ} 14^{\prime} 24^{\prime \prime} \mathrm{W}$ & 454 & Aw \\
\hline Oriente/SP & $22^{\circ} 09^{\prime} 15^{\prime \prime}$ S e $50^{\circ} 05^{\prime} 35^{\prime \prime} \mathrm{W}$ & 599 & Aw \\
\hline Urupês/SP & $21^{\circ} 12^{\prime} 02^{\prime \prime}$ S e $49^{\circ} 17^{\prime} 25^{\prime \prime} \mathrm{W}$ & 440 & Aw \\
\hline Ariranha/SP & $21^{\circ} 11^{\prime} 23^{\prime \prime}$ S e $48^{\circ} 47^{\prime} 17^{\prime \prime} \mathrm{W}$ & 585 & Aw \\
\hline Brejinho de Nazaré/T0 & $11^{\circ} 01^{\prime} 06^{\prime \prime}$ S e $48^{\circ} 34^{\prime} 08^{\prime \prime} W$ & 239 & Aw \\
\hline Timbaúba/PE & $07^{0} 30^{\prime} 38^{\prime \prime}$ S e $35^{\circ} 19^{\prime} 06^{\prime \prime} \mathrm{W}$ & 107 & As \\
\hline Juazeiro/BA & $09^{\circ} 29^{\prime} 59^{\prime \prime} \mathrm{S}$ e $40^{\circ} 22^{\prime} 05^{\prime \prime} \mathrm{W}$ & 398 & Bswh \\
\hline Chapada dos Guimarães/MT & $14^{\circ} 59^{\prime} 39^{\prime \prime}$ S e $55^{\circ} 49^{\prime} 07^{\prime \prime} W$ & 350 & Aw \\
\hline Jaíba/MG & $15^{\circ} 20^{\prime} 23^{\prime \prime}$ S e $43^{\circ} 40^{\prime} 39^{\prime \prime} \mathrm{W}$ & 480 & Bswh \\
\hline São João de Pirabas/PA & $00^{\circ} 47^{\prime} 12^{\prime \prime}$ S e $47^{\circ} 10^{\prime} 59^{\prime \prime} \mathrm{W}$ & 22 & Ami \\
\hline
\end{tabular}


Tabela 2. Dados de $\mathrm{pH}$, macronutrientes e matéria orgânica na profundidade de $0-20 \mathrm{~cm}$ de solos localizados sob plantios de nim, em vários municípios do Brasil

\begin{tabular}{|c|c|c|c|c|c|c|c|c|}
\hline \multirow{2}{*}{ Local de plantio } & \multirow{2}{*}{$\begin{array}{c}\mathrm{pH} \\
\mathrm{CaCl}_{2}\end{array}$} & K & $\mathrm{Ca}$ & $\mathrm{Mg}$ & $\mathrm{Al}$ & $\mathrm{H}+\mathrm{Al}$ & M.0 & \multirow{2}{*}{$\underset{\mathrm{mg} \mathrm{dm^{-3 }}}{P}$} \\
\hline & & \multicolumn{6}{|c|}{$\mathrm{cmol}_{\mathrm{c}} \mathrm{dm}^{-3}$} & \\
\hline Xambrê/PR & 4,9 & 0,18 & 1,36 & 0,16 & 0,00 & 5,28 & 10,79 & 3,7 \\
\hline lbirá/SP & 4,1 & 0,30 & 1,28 & 0,38 & 0,52 & 6,13 & 6,90 & 1,8 \\
\hline Oriente/SP & 5,3 & 0,11 & 1,80 & 0,50 & 0,00 & 1,60 & 9,00 & 15,0 \\
\hline Urupês/SP & 5,4 & 0,44 & 2,50 & 1,74 & 0,00 & 3,69 & 8,17 & 2,9 \\
\hline Timbaúba/PE & 5,4 & 0,33 & 7,35 & 2,60 & 0,00 & 3,31 & 34,78 & 46,8 \\
\hline Juazeiro/BA & 7,0 & 1,00 & 4,83 & 1,07 & 0,00 & 1,25 & 22,25 & 27,5 \\
\hline Chapada dos Guimarães/MT & 4,1 & 0,35 & 1,97 & 1,02 & 0,60 & 3,76 & 2,99 & 1,1 \\
\hline Jaíba/MG & 5,8 & 0,45 & 2,12 & 1,35 & 0,02 & 1,61 & 8,51 & 16,2 \\
\hline São João de Pirabas/PA & 4,4 & 0,08 & 1,16 & 0,38 & 0,36 & 3,19 & 15,22 & 5,4 \\
\hline
\end{tabular}

Tabela 3. Local e tipo de solo sob plantios de nim, em diferentes estados do Brasil

\begin{tabular}{ll}
\hline \multicolumn{1}{c}{ Local de plantio/estado } & Tipo de solo \\
Londrina/PR & Latossolos Vermelho eutroférricos com horizonte A moderado, textura argilosa e relevo suave ondulado \\
Xambrê/PR & Argissolos Vermelho-Amarelo Eutrófico com horizonte A moderado, textura arenosa e relevo suave ondulado \\
Jales/SP & Argissolos Vermelho-Amarelo Eutrófico com horizonte A moderado, textura arenosa e relevo suave ondulado \\
lbirá/SP, Oriente/SP, Urupês/SP, Ariranha/SP & Argissolos Vermelho-Amarelos Eutrófico com horizonte A abrúptico, textura arenosa e relevo suave ondulado \\
Brejinho de Nazaré/TO & Latossolos Vermelho-Amarelo Distróficos plínticos, com baixa saturação de bases, relevo suave ondulado \\
Timbaúba/PR & Luvissolos Crômicos Órticos planossólicos e Neossolos Litólicos eutro -úmbricos \\
Juazeiro/BA & Argissolos Amarelo Eutrófico, concrecionário, textura arenosa, relevo plano \\
Chapada dos Guimarães/MT & Neossolos Quartzarênico Órticos, textura arenosa e relevo suav e ondulado \\
Jaíba/MG & Latossolos Amarelo Distróficos típicos, textura arenosa e relevo suave ondulado \\
São João de Pirabas/PA & Argissolos Amarelo Distróficos, textura média e relevo suave ondulado \\
\hline
\end{tabular}
Fonte: EMBRAPA (2006)

Tabela 4. Teores médios de N, P, K, Ca e Mg $\left(\mathrm{g} \mathrm{kg}^{-1}\right)$ nas FCMS e FCMI de nim, em plantios localizados em diferentes municípios do Brasil

\begin{tabular}{|c|c|c|c|c|c|c|c|c|c|c|c|}
\hline \multirow{2}{*}{$\begin{array}{c}\text { Local de } \\
\text { plantio }\end{array}$} & \multirow{2}{*}{$\begin{array}{l}\text { Idade do } \\
\text { plantio } \\
\text { ano }\end{array}$} & \multicolumn{5}{|c|}{ FCMS } & \multicolumn{5}{|c|}{ FCMI } \\
\hline & & $\mathrm{N}$ & $\mathbf{P}$ & K & $\mathrm{Ca}$ & $\mathrm{Mg}$ & $\mathbf{N}$ & $\mathbf{P}$ & $\mathbf{K}$ & $\mathrm{Ca}$ & $\mathrm{Mg}$ \\
\hline Londrina/PR & 5 & $\star 34,83 \mathrm{bc}$ & $2,00 \mathrm{~cd}$ & $24,72 \mathrm{a}$ & *5,78 h & $\star 2,22 f$ & 28,72 bc & $1,84 \mathrm{c}$ & $24,06 \mathrm{a}$ & $11,33 \mathrm{~h}$ & $3,67 \mathrm{c}$ \\
\hline Jales/SP & 2,5 & *38,11 a & *2,39 c & *16,94 de & ${ }^{\star} 6,50 \mathrm{~h}$ & $\star 3,61$ e & $31,33 \mathrm{a}$ & $1,80 \mathrm{c}$ & $11,22 \mathrm{~g}$ & $25,33 \mathrm{e}$ & $7,28 \mathrm{a}$ \\
\hline lbirá/SP & 3,5 & $\star 33,72 \mathrm{c}$ & $\star 2,28 \mathrm{c}$ & $\star 21,28 \mathrm{~b}$ & *15,94 e & ${ }^{*} 4,50 \mathrm{~cd}$ & $31,28 a$ & $1,56 \mathrm{~cd}$ & $18,06 \mathrm{~b}$ & $22,39 f$ & $5,22 b$ \\
\hline Oriente/SP & 4 & $\star 29,33$ de & $\star 5,00 \mathrm{a}$ & $\star 15,83$ ef & $\star 28,00 \mathrm{~b}$ & $\star 5,17 \mathrm{bc}$ & $24,61 d$ & $3,61 \mathrm{ab}$ & $11,89 \mathrm{fg}$ & $50,44 a$ & $7,50 \mathrm{a}$ \\
\hline Brejinho de Nazaré/T0 & 5 & $26,44 f$ & $\star 2,22 \mathrm{c}$ & $* 15,44 \mathrm{fg}$ & *10,67 g & $3,50 \mathrm{e}$ & $26,00 \mathrm{~d}$ & $1,83 \mathrm{c}$ & 13,11 ef & $15,11 \mathrm{~g}$ & $3,61 \mathrm{c}$ \\
\hline Timbaúba/PE & 6 & $\star 30,67 d$ & $\star 1,11 \mathrm{e}$ & $\star 17,89 \mathrm{~d}$ & $\star 24,83 \mathrm{c}$ & $\star 6,11 \mathrm{a}$ & $29,11 \mathrm{abc}$ & $0,61 f$ & $16,28 \mathrm{~cd}$ & $27,89 \mathrm{~d}$ & $6,28 \mathrm{~b}$ \\
\hline Juazeiro/BA $^{1}$ & 7 & *18,94 g & $\star 1,22 \mathrm{e}$ & $* 14,44 \mathrm{~g}$ & $\star 30,72 \mathrm{a}$ & $5,06 \mathrm{bc}$ & $15,56 \mathrm{e}$ & 1,00 ef & $13,78 \mathrm{e}$ & $38,78 \mathrm{~b}$ & $5,33 b$ \\
\hline Chapada dos Guimarães/MT & 5 & $\star 30,67 \mathrm{~d}$ & $\star 1,56 \mathrm{de}$ & $8,78 \mathrm{~h}$ & *10,39 g & $3,22 \mathrm{e}$ & $28,22 \mathrm{c}$ & $1,22 \mathrm{de}$ & $8,56 h$ & $13,44 \mathrm{~g}$ & $3,72 \mathrm{c}$ \\
\hline Jaíba/MG & 2,5 & *39,11 a & *4,67 a & $5,39 \mathrm{i}$ & $* 22,39 \mathrm{~d}$ & $\star 4,78 \mathrm{c}$ & $29,44 a b c$ & $4,00 \mathrm{a}$ & $5,22 j$ & $35,22 \mathrm{c}$ & $5,89 \mathrm{~b}$ \\
\hline São João de Pirabas/PA & 2,5 & $\star 30,83 d$ & $* 4,11 \mathrm{~b}$ & $7,50 \mathrm{~h}$ & $* 13,67 \mathrm{f}$ & $3,22 \mathrm{e}$ & $24,78 d$ & $3,17 \mathrm{~b}$ & $6,94 \mathrm{i}$ & $25,44 \mathrm{e}$ & $4,00 \mathrm{C}$ \\
\hline
\end{tabular}

FCMS - Folha da copa média superior; FCMI - Folha da copa média inferior; * médias nas linhas diferem entre si pelo Teste de Duncan ( $\mathrm{p} \leq 0,05)$; médias nas colunas seguidas da mesma letra não diferem entre sí pelo Teste de Duncan ( $\leq \leq 0,05)$; CV - coeficiente de variação; ${ }^{1}$ Plantio com fertirrigação

não evidenciaram diferença significativa para os mencionados nutrientes.

Entre os diferentes locais de plantio os maiores e menores teores médios $\left(\mathrm{g} \mathrm{kg}^{-1}\right)$ obtidos foram: para N nas FCMS (39,11; 38,11 ), em Jaíba/Mg e Jales/SP, respectivamente, e nas FCMI $(31,33 ; 15,56)$, em Jales/SP e Juazeiro/BA, respectivamente. Para P nas FCMS $(5,00 ; 4,67)$, em Oriente/SP e Jaíba/MG, respectivamente, e nas FCMI $(4,00 ; 0,61)$, em Jaíba/MG e Timbaúba/PE, respectivamente. Para K nas FCMS $(24,72$; $5,39)$ e nas FCMI $(24,06 ; 5,22)$, em Londrina/PR e Jaíba/MG, respectivamente. Para Ca nas FCMS $(30,72 ; 5,78)$, em Juazeiro/
BA e Londrina/PR, respectivamente, e nas FCMI $(50,44 ; 38,78)$, em Oriente/SP e Juazeiro/BA, respectivamente. Para Mg $(6,11$; 2,22), em Timbaúba/PE e Londrina/PR, respectivamente, e nas FCMI $(7,50 ; 3,61)$, em Oriente/SP e Brejinho de Nazaré/TO, respectivamente (Tabela 4).

A escolha do melhor terço da copa de árvores para amostragem foliar continuará sendo assunto aberto a discussões visto que mesmo na parte mediana se verificam variações quando esta porção da copa é subdividia (Tabela 4). Normalmente, quando a amostragem foliar é feita em três partes da copa (superior, média e inferior) a tendência é que os teores 
dos nutrientes considerados de pouca mobilidade, a exemplo do $\mathrm{Ca}$, se acumulem na parte inferior (Evans, 1979; Souza et al., 2008), enquanto os de N, P, Zn e B diminuem (Evans, 1979).

Há carência de informações na literatura sobre a amostragem de folhas de nim, no que diz respeito à definição da posição das folhas na copa das árvores. Segundo Evans (1979) este tema é bastante controverso. Este autor menciona que a posição do topo da copa é, provavelmente, a escolha mais segura pelo fato de ser a menos sombreada. Porém Lima et al. (2007) mencionam que a coleta de folhas quando feita no terço médio representa um aspecto bastante concreto da amostragem. Nesta posição da copa a acessibilidade ao material requerido é melhor e também apresenta melhor luminosidade do que o material situado na parte inferior da copa visto que o sombreamento influencia decisivamente na concentração dos elementos (Evans, 1979; Insley et al., 1981) e nos aspectos do metabolismo e da morfologia foliar (Rossato et al., 2010).

Referente aos os resultados apresentados na Tabela 4, podese afirmar que elementos minerais como $\mathrm{N}, \mathrm{P} \mathrm{e} \mathrm{K}$, considerados móveis nas plantas (Hawkesford et al., 2012) apresentam, como regra, maiores concentrações nas folhas fisiologicamente mais ativas enquanto para $\mathrm{Ca}$, nutriente considerado de baixa mobilidade na planta, as folhas velhas são as que apresentam os maiores teores deste nutriente (Epstein \& Bloom, 2006). $\mathrm{O}$ resultado obtido com o $\mathrm{Mg}$ pode ser atribuído ao tipo de metodologia usada para coleta do material vegetal. Evans (1979) relata que este nutriente é, dentre outros, notadamente o que apresenta constante variação em amostragem foliar dentro da copa.

Além da mobilidade dos nutrientes anteriormente mencionados os resultados na Tabela 4 podem ser atribuídos também à luminosidade, visto que o sombreamento influencia de forma decisiva nos teores dos nutrientes (Evans, 1979; Insley et al., 1981) aos aspectos do metabolismo e da morfologia foliar (Rossato et al., 2010) e da região de crescimento (Sabaté et al., 1995). Também há de se considerar que a espécie em questão é altamente exigente em luminosidade (Puri \& Swamy, 2001).

Analisando nutricionalmente a Tabela 4, chama-se a atenção para os teores obtidos de N (FCMS) e Ca (FCMS e FCMI). Ambos se situam no limite superior máximo do intervalo de concentração reportado na literatura para plantas cultivadas (Epstein \& Bloom, 2006).

Os resultados obtidos com N (Tabela 4) são expressivos dentro da nutrição mineral florestal e, também, da exigência do nim com relação à água, visto que os plantios em Juazeiro são conduzidos com fertirrigação, apesar de ser uma espécie com tolerância ao estresse hídrico (Puri \& Swamy, 2001). Esses resultados merecem ser melhor estudados visto que parecem muito expressivos e de relevância para fins práticos; como exemplo, pode-se mencionar, mediante observações de campo feitas em árvores solteiras, por autores deste trabalho, que se constatou que as mesmas, quando cultivadas sobre solos de fertilidade natural baixa em clima seco, próximas de dejetos orgânicos depositados pelo homem ou de criações de animais (suínos) evidenciaram bom crescimento com copas largas e frondosas e, em consequência, boa produção de frutos.

Os resultados obtidos com o $\mathrm{N}$ também podem ser atribuídos ao fato do nim ser eficiente na absorção deste nutriente mesmo quando há limitação de oferta de $\mathrm{N}$ no solo, desde que luz e P não sejam limitantes (Puri \& Swamy, 2001). Neste caso, ressalta-se que no presente trabalho esses fatores não são limitantes, tanto para P (Tabela 2) quanto para luz, devido ao fato de que os plantios amostrados foram estabelecidos a pleno sol.

Quanto ao $\mathrm{Ca}$, os teores encontrados nas folhas (Tabela 4) remetem a uma planta de característica calcícola, visto que aproximadamente $50 \%$ se enquadram na classificação proposta por How \& Merkle (1950) que os classificam como calcícolas, espécies que apresentam teores foliares de Ca na ordem de 15,7 a $22,7 \mathrm{~g} \mathrm{~kg}^{-1}$, enquanto nas calcífugas esses teores se situam no intervalo de 4,8 a 10,8 $\mathrm{g} \mathrm{kg}^{-1}$.

Em um estudo desenvolvido na região de Uberlândia, MG, em duas áreas de florestas com espécies lenhosas nativas, foram consideradas calcícolas as espécies que ocorriam exclusivamente em solo eutrófico com elevados teores de $\mathrm{Ca}$ e menor acidez, condições que contribuíram para que $30 \%$ das espécies estudadas apresentassem teores foliares de $\mathrm{Ca}$ superiores a 14,8 $\mathrm{g} \mathrm{kg}^{-1}$ (Haridasan \& Araújo, 2005). Conforme se observa na Tabela 2, os teores de $\mathrm{Ca}$ nos solos amostrados predominam na faixa considerada de baixa a média (SBCS, 2004). Portanto, os elevados teores de $\mathrm{Ca}$ encontrados nas duas posições das copas amostradas (Tabela 4) evidenciam uma capacidade de absorção do elemento pelo nim, não condizente com os teores no solo (Tabela 2) podendo tal fato estar associado a uma elevada CTC das raízes (Hanson, 1984).

A obtenção dos elevados teores de cálcio existentes nas folhas de nim pode ser explicada, em parte, devido ao fato deste nutriente ser absorvido por fluxo de massa (Ruiz et al., 1999; Miotto, 2009) o que representa, para a planta, condição de plena disponibilidade deste macronutriente (Miotto, 2009). Este autor ressalta que a diminuição do teor de água no solo implica no aumento da concentração do cálcio na solução do solo podendo manter um suprimento adequado do nutriente à planta, mesmo quando a umidade do solo não esteja em níveis ótimos. Desta forma, o $\mathrm{Ca}$ poderia ter acesso à parte aérea mediante entrada apoplástica das extremidades radiculares sem estrias de Caspari e ainda não suberizadas (Epstein \& Bloom, 2006) embora o mecanismo não dependa unicamente do fluxo de massa mas também de outros processos de troca, em virtude do Ca ser fortemente adsorvido em sítios negativos (Epstein \& Bloom, 2006). Este assunto é bastante controverso visto que ainda não foi suficientemente elucidado (Yang \& Jie, 2005).

Os aspectos anteriormente mencionados à absorção do Ca remetem a uma análise para os teores encontrados para K (Tabela 4) visto que, segundo Nordstrom (1982) a CTC das raízes varia sazonalmente interferindo, desta forma, na relação K/Ca da parte aérea. No caso do nim deve-se comentar que no presente trabalho a maioria das amostras foi coletada durante o outono (abril). Porém, considerando-se uma única estação de coleta os resultados obtidos (Tabela 4) mostram haver alternância na relação $\mathrm{K} / \mathrm{Ca}$, ou seja, ora existindo favorecimento ao $\mathrm{K}$ e ora favorecendo o $\mathrm{Ca}$. Isto pode significar que tanto para a CTC ou para o fluxo transpiratório a relação desses dois nutrientes estaria subordinada às condições locais de amostragem cujo resultado mostra a necessidade de se aprofundar o conhecimento sobre esta relação para a espécie 
em questão mas ao mesmo tempo reforça os indícios de ser o nim uma planta calcícola.

Na revisão bibliográfica sobre teores de macronutrientes em folhas de nim encontrou-se apenas o trabalho de revisão sobre aspectos nutricionais de várias espécies tropicais e subtropicais conduzido por Drechsel \& Zech (1991). Na Tabela 5 se apresentam os teores foliares de $\mathrm{N}, \mathrm{P}, \mathrm{K}, \mathrm{Ca}$ e $\mathrm{Mg}$ nas faixas consideradas deficiente, baixo e satisfatório para o nim.

Tabela 5. Teores foliares $\left(\mathrm{g} \mathrm{kg}^{-1}\right)$ de N, P, K, Ca e Mg considerados deficientes, baixos e satisfatórios em plantios de nim, com diferentes idades

\begin{tabular}{|c|c|c|c|c|c|}
\hline \multirow{2}{*}{ Espécie } & \multirow{2}{*}{ Nutriente } & \multirow{2}{*}{$\begin{array}{l}\text { Idade } \\
\text { (anos) }\end{array}$} & \multicolumn{3}{|c|}{ Teores $\left(\mathrm{g} \mathrm{kg}^{-1}\right)$} \\
\hline & & & Deficiente & Baixo & Satisfatório \\
\hline \multirow{9}{*}{$\mathrm{Nim}$} & $\mathrm{N}$ & $1-6$ & - & $12,6-15,6$ & $19,6-28,0$ \\
\hline & & $1-6$ & - & $0,9-1,1$ & $1,1-1,5$ \\
\hline & $P$ & 10 & - & 1,2 & 1,4 \\
\hline & & 1 & 1,7 & - & 6,5 \\
\hline & K & $2-6$ & - & - & $9,9-17,9$ \\
\hline & & 10 & - & $1,5-1,8$ & - \\
\hline & $\Gamma$ & 1,4 & - & - & $14,0-23,2$ \\
\hline & va & $2-6$ & - & - & $17,1-35,5$ \\
\hline & $\mathrm{Mg}$ & $1-10$ & - & - & $2,4-6,0$ \\
\hline
\end{tabular}

Fonte: adaptado de Drechsel \& Zech (1991)

Os teores foliares mencionados na Tabela 4 permitem indicar, quando comparados com os apresentados na Tabela 5 , que os teores existentes nos diferentes solos (Tabela 2) não foram satisfatórios para suprir a demanda do nim nos plantios localizados apenas em Timbaúba e Juazeiro para P; no entanto e segundo os dados da Tabela 2, são considerados muito altos os teores de $\mathrm{P}$ nos solos desses dois locais. Adicionalmente, os teores de matéria orgânica são bastante altos o que, em princípio, ofereceria boa condição para aproveitamento do fósforo orgânico (Achat et al., 2009). Por outro lado teores de P em solos de outros seis locais podem ser considerados baixos, até extremamente baixo em Brejinho de Nazaré, sem que os teores de P nas folhas de nim sejam tidos como não satisfatórios, o que pode evidenciar a influência de outros fatores na absorção deste nutriente pela planta já que o $\mathrm{P}$ do solo com o $\mathrm{P}$ foliar nas duas posições da copa amostrada não estão correlacionados (Tabela 6 e Figuras 1A e 1B).

Para K, os teores nos solos dos diferentes locais podem ser considerados de médios a altos e em apenas um local (Jaíba) o teor na folha é tido como não satisfatório. O mesmo ocorre
A.

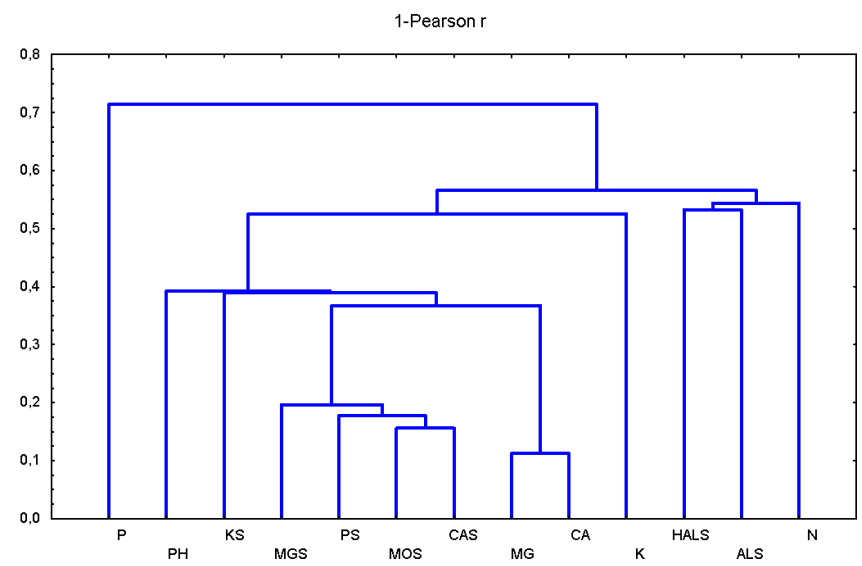

B.

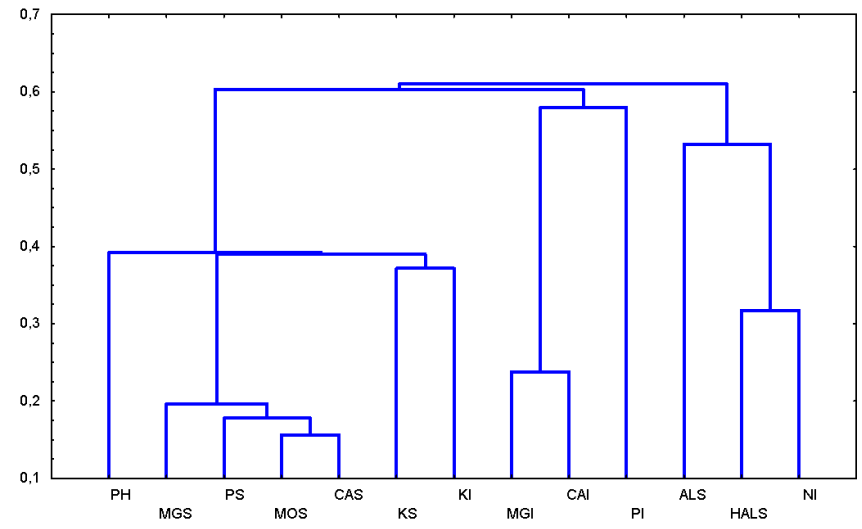

Figura 1. Análise de grupamento para atributos dos solos e nutrientes da planta na FCMS de nim (1-Pearson, $p \leq 0,05)$. Variáveis com índice "S" representam atributos solo e variáveis sem o índice representam os nutrientes da copa (B)

com os teores de Ca nos solos, passíveis de ser considerados de médios a altos mas nas folhas de nim de seis locais os teores deste elemento não são satisfatórios.

Para o Mg, os teores nos solos dos diferentes locais são considerados de médios a altos, com exceção de quatro deles; apesar disto, nas folhas de nim apenas em um local os teores não são satisfatórios. Do exposto, pode-se constatar que enquanto alguns solos apresentam teores baixos de algum nutriente nas

Tabela 6. Correlações entre teores de $\mathrm{pH}, \mathrm{P}, \mathrm{K}, \mathrm{Ca}, \mathrm{Mg}, \mathrm{Al}, \mathrm{H}+\mathrm{Al}$ e $\mathrm{MO}$ no solo e de $\mathrm{N}, \mathrm{P}, \mathrm{K}, \mathrm{Ca}$ e $\mathrm{Mg}$ existentes nas folhas em posição da folha da copa média superior (FCMS) e da folha da copa média inferior (FCMI) de nim

\begin{tabular}{|c|c|c|c|c|c|c|c|c|c|}
\hline \multirow{2}{*}{$\begin{array}{l}\text { Posição } \\
\text { da folha }\end{array}$} & \multirow{2}{*}{$\begin{array}{c}\text { Teores } \\
\text { nas } \\
\text { folhas }\end{array}$} & \multicolumn{8}{|c|}{ Características químicas dos solos } \\
\hline & & pH & $\mathbf{P}$ & K & $\mathrm{Ca}$ & $\mathrm{Mg}$ & Al & $\mathrm{H}+\mathrm{Al}$ & MO \\
\hline \multirow{4}{*}{ FCMS } & $\mathrm{N}$ & $-0,35$ & $-0,31$ & $-0,01$ & $-0,32$ & 0,02 & 0,13 & 0,46 & $-0,47$ \\
\hline & $\mathrm{K}$ & 0,22 & 0,01 & 0,47 & 0,40 & 0,34 & $-0,22$ & 0,43 & 0,18 \\
\hline & $\mathrm{Ca}$ & 0,35 & $0,62^{\star}$ & $-0,19$ & 0,32 & $-0,04$ & $-0,45$ & $-0,61^{*}$ & 0,48 \\
\hline & $\mathrm{Mg}$ & 0,16 & $0,63^{*}$ & $-0,37$ & 0,34 & 0,01 & 0,34 & $-0,38$ & 0,49 \\
\hline \multirow[t]{3}{*}{ FCMI } & K & 0,31 & 0,06 & $0,63^{*}$ & 0,54 & 0,47 & $-0,29$ & 0,39 & 0,31 \\
\hline & $\mathrm{Ca}$ & 0,28 & 0,40 & $-0,30$ & 0,00 & $-0,26$ & $-0,42$ & $-0,64^{\star}$ & 0,15 \\
\hline & $\mathrm{Mg}$ & 0,11 & 0,36 & $-0,30$ & 0,07 & $-0,11$ & $-0,19$ & $-0,35$ & 0,04 \\
\hline
\end{tabular}

FCMS - Folha da copa média superior; FCMI - Folha da copa média inferior; ${ }^{\star} \mathrm{p} \leq 0,05$ 
Tabela 7. Correlações entre teores de $\mathrm{pH}, \mathrm{P}, \mathrm{K}, \mathrm{Ca}, \mathrm{Mg}, \mathrm{Al}, \mathrm{H}+\mathrm{Al}$ e $\mathrm{MO}$ nos solos com plantios de nim

\begin{tabular}{|c|c|c|c|c|c|c|c|}
\hline \multirow{2}{*}{$\begin{array}{c}\text { Variáveis } \\
\text { do solo }\end{array}$} & $\mathbf{P}$ & K & $\mathrm{Ca}$ & $\mathrm{Mg}$ & Al & $\mathrm{H}+\mathrm{Al}$ & MO \\
\hline & \multicolumn{7}{|c|}{$r$} \\
\hline pH & 0,46 & $0,60^{*}$ & $0,61^{*}$ & 0,50 & $-0,76^{* *}$ & $-0,61^{*}$ & 0,46 \\
\hline $\mathrm{P}$ & - & 0,13 & $0,76^{\star \star}$ & 0,54 & $-0,43$ & $-0,46$ & $0,82^{\star \star}$ \\
\hline K & & - & $0,57^{*}$ & $0,61^{*}$ & $-0,27$ & $-0,04$ & 0,22 \\
\hline $\mathrm{Ca}$ & & & - & $0,80^{* *}$ & $-0,49$ & $-0,25$ & $0,84^{* *}$ \\
\hline $\mathrm{Mg}$ & & & & - & $-0,39$ & $-0,18$ & 0,54 \\
\hline $\mathrm{Al}$ & & & & & - & 0,47 & $-0,51$ \\
\hline $\mathrm{H}+\mathrm{Al}$ & & & & & & - & $-0,27$ \\
\hline
\end{tabular}

${ }^{\star *} p \leq 0,01 ;{ }^{*} p \leq 0,05$

folhas tais deficiências dificilmente são observadas e nem sempre são coincidentes.

A análise estatística mostrou haver correlação positiva significativa $(\mathrm{p} \leq 0,05)$ entre os valores de $\mathrm{P}$ do solo com os teores de $\mathrm{Ca}$ e $\mathrm{Mg}$ nas folhas da posição FCMS; as correlações foram negativas entre os valores de $\mathrm{H}+\mathrm{Al}$ do solo e os teores de Ca nas folhas, em ambas as posições; já o teor de $\mathrm{K}$ no solo se correlacionou positivamente com o teor de $\mathrm{K}$ na folha da posição $\mathrm{FCMI}$ e correlação positiva de $\mathrm{H}+\mathrm{Al}$ do solo com os teores de $\mathrm{N}$ da folha na posição da FCMI de nim (Tabela 6), sendo esta de difícil explicação em virtude do alcance metodológico utilizado na condução do trabalho. Talvez uma análise discriminada do nitrogênio do solo, nas suas formas iônicas, nítrica/amoniacal, seria mais elucidativa.

Nas Figuras 1A e 1B se apresentam os resultados da análise de agrupamento (1-Pearson r) para os teores das FCMS e FCMI.

Observa-se que os grupamentos constituídos corroboram com o exposto na matriz de correlação (Tabela 7). É importante destacar que na Figura 1A a relação $\mathrm{Ca}$ e Mg da FCMS com o P do solo está de acordo com a matriz de correlação (Tabela 7) observando-se a ligação existente do P e Ca com a M.O. No caso específico da correlação positiva entre P e M.O. do solo é importante frisar a elevada importância do P-orgânico para o P disponível no solo (Achat et al., 2009). Por outro lado, nota-se que na Tabela 6 e nas Figuras 1A e 1B o N da planta e a matéria orgânica (M.O) do solo não são correlacionados.

Com a correlação positiva entre K do solo com o K da FCMI (Tabela 6) quando visualizada na análise de grupamento (Figura 1B) percebe-se que a mesma representa com propriedade a relação existente entre os atributos do solo e a nutrição da planta.

\section{Conclusões}

1. Tendo em vista a alta mobilidade dos elementos na planta, como o N, e a baixa mobilidade, a exemplo do $\mathrm{Ca}$, é preciso considerar com propriedade a posição de amostragem (folhas superiores e/ou inferiores) na interpretação dos resultados.

2. Os elevados teores foliares de $\mathrm{Ca}$ encontrados nas duas posições das copas amostradas apresentam indícios de o nim ser uma espécie calcícola.

3. Independente dos teores dos nutrientes nos solos amostrados, o nim evidencia ser uma espécie eficiente na absorção de N, P e Ca.

4. Dos nutrientes estudados apenas o K apresentou correlação positiva entre seu teor no solo e o teor nas folhas da posição da copa média/inferior.

\section{LITERATURA CITADA}

Achat, D. L.; Bakker, M. R; Morel, C. Process-based assessment of phosphorus availability in a low phosphorus sorbing forest soil using isotopic dilution methods. Soil Science Society American of Journal, v.73, p.2131-2142, 2009.

Drechsel, P.; Zech, W. Foliar nutrient levels of broad-leaved tropical trees: A tabular review. Plant and Soil, v.131, p.29-46, 1991.

EMBRAPA - Empresa Brasileira de Pesquisa Agropecuária. Sistema brasileiro de classificação de solos. 2.ed. Brasília: Embrapa Informação Tecnológica; Rio de Janeiro: Embrapa Solos, 2006. 306p.

Epstein, E.; Bloom, A. J. Nutrição mineral de plantas: Princípios e perspectivas. Londrina: Planta, 2006. 403p.

Evans, J. The effects of leaf position and leaf age in foliar analysis of Gmelina arborea. Plant and Soil, v. 2, p.547$552,1979$.

Girish, K.; Bhat, S. S. Neem - A green treasure. Electronic Journal of Biology, v.4, p.102-111, 2008.

Hanson, J. B. The function of calcium in plant nutrition. In: Tinker, P. B.; Laeuchli, A. (ed.). Advances in Plant nutrition. v.1, New York: Praeger Publishers, 1984. p.149- 208.

Haridasan, M.; Araújo, G. M. Perfil nutricional de espécies lenhosas de duas florestas semidecíduas em Uberlânida, MG. Revista Brasileira de Botânica, v.28, p.295-303, 2005.

Hawkesford, M.; Horst, W.; Kichey, T.; Lambers, H.; Schjoerring, J.; Moller, S. I.; White, P. Functions of macronutrients. In: Marschner, P. (ed.). Marschner's mineral nutrition of higher plants. NewYork: Elsevier, 2012. cap.6, p.135-189.

How, H. Y.; Merkle, F. G. Chemical composition of certain calcifugous and calcicole plants. Soil Science, v.69, p.471486, 1950.

Insley, H.; Boswell, R. C.; Gardiner, J. B. H. Foliar macronutrients (N, P, K, Ca and Mg) in lime (Tilia sp.). I Sampling techniques. Plant and Soil, v.61, p.377-389, 1981.

Lima, R. de L. S. de; Ferreira, G. B.; Weber, O. B.; Cazetta, J. O. Diagnose foliar da gravioleira (Annona muricata L.): Efeito da posição de ramos e folhas. Ciência e Agrotecnologia, v.31, p.1320-1325, 2007.

Maithani, A.; Parcha, V.; Pant, G.; Dhulia, I.; Kumar, D. Azadirachta indica (neem) leaf: A review. Journal of Pharmacy Research, v.4, p.1824-1827, 2011.

Miotto, A. Calcários calcíticos e dolomíticos e alterações nos atributos de solos e plantas sob sistema de plantio direto. Santa Maria: UFSM, 2009. 78p. Dissertação Mestrado 
Nogueira, A. R. de A.; Souza, G. B. de (ed.). Manual de laboratório: Solo, água, nutrição vegetal, nutrição animal e alimentos. São Carlos: Embrapa Pecuária Sudeste, 2005. $313 p$.

Nordstrom, I. O. Variablility between species in K/Ca rations of successional plants. Plant and Soil, v.65, p.137-139, 1982.

Pankaj, S,; Lokeshwar, T.; Mukesh, B.; Vishnu, B.; Review on neem (Azadirachta indica): Thousand problems one solution. International Research Journal of Pharmacy, v.2, p.97-102, 2011.

Puri, S.; Swamy, S. L. Growth and biomass production in Azadirachta indica seedlings in response to nutrients (N and P) and moisture stress. Agroforestry Systems, v.51, p.57-68, 2001.

Rossato, D. R.; Takahashi, F. S. C.; Silva, L. de C. R.; Franco, A. C. Características funcionais de folhas de sol e sombra de espécies arbóreas em um mata de galeria no Distrito Federal, Brasil. Acta Botanica Brasilica, v.24, p.640-647, 2010.
Ruiz, H. A.; Miranda, J.; Conceição, J. C. S. Contribuição dos mecanismos de fluxo de massa e de difusão para o suprimento de $\mathrm{K}, \mathrm{Ca}$ e $\mathrm{Mg}$ a plantas de arroz. Revista Brasileira de Ciência do Solo, v.23, p.1015-1018, 1999.

Sabaté, S.; Sala, A.; Gracia, C. A. Nutrient content in Quercus ilex canopies: Seasonal and spatial variation within a catchment. Plant and Soil, v.168/169, p.297-307, 1995.

SBCS - Sociedade Brasileira de Ciência do Solo. Manual de adubação e de calagem para os estados do Rio Grande do Sul e de Santa Catarina. 10.ed. Porto Alegre: SBCS, Comissão de química e fertilidade do solo, 2004. 400p.

Souza, J. L. M. de.; Araujo, M. A. de; Reissmann, C. B.; Maccari Júnior, A.; Wolf. C. S. Teores de nutrientes foliares em plantas de erva-mate em função da posição e orientação geográfica da copa, em Guarapuava-PR. Scientia Agraria, v.9, p.49-58, 2008.

Yang, Hong-Qiang.; Jie, Yu-Ling. Uptake and transport of calcium in plants. Journal of Plant Physiology and Molecular Biology, v.31, p.227-234, 2005. 